\title{
E-cigarettes versus nicotine patch for perioperative smoking cessation: a pilot randomized trial
}

\author{
Susan M Lee ${ }^{\text {Corresp., } 1,2,3,4}$, Rachel Tenney ${ }^{1,2}$, Arthur W Wallace ${ }^{1,2}$, Mehrdad Arjomandi ${ }^{5}$ \\ 1 Department of Anesthesia and Perioperative Care, University of California, San Francisco, San Francisco, California, United States of America \\ 2 Anesthesia Service, San Francisco Veterans Affairs Medical Center, San Francisco, California, United States of America \\ 3 \\ 4 Department of Anesthesia, Royal Columbian Hospital, New Westminster, British Columbia, Canada \\ 5 Department of Pulmonary and Critical Care Medicine, San Francisco Veterans Affairs Medical Center, San Francisco, California, United States of America \\ Corresponding Author: Susan M Lee \\ Email address: susanlee.anesthesia@gmail.com
}

Introduction: Cigarette smoking by surgical patients is associated with increased complications. Ecigarettes have emerged as a potential smoking cessation tool. We sought to determine the feasibility and acceptability of e-cigarettes, compared to nicotine patch, for perioperative smoking cessation in veterans.

Methods: Preoperative patients were randomized to either the nicotine patch group $(n=10)$ or the ecigarette group $(n=20)$. Both groups were given a free 6 -week supply in a tapering dose. All patients received brief counseling, a brochure on perioperative smoking cessation, and referral to the California Smokers' Helpline. The primary outcome was rate of smoking cessation on day of surgery confirmed by exhaled carbon monoxide. Secondary outcomes included smoking habits, pulmonary function, adverse events, and satisfaction with the products on day of surgery and at 8-weeks follow-up.

Results: Biochemically verified smoking cessation on day of surgery was similar in both groups. Change in forced expiratory volume in one second (FEV1) was $592 \mathrm{ml}$ greater in the e-cigarette group $(95 \% \mathrm{Cl}$ $153-1031 \mathrm{ml}, \mathrm{p}=0.01$ ) and change in forced expiratory volume in one second to forced vital capacity ratio (FEV1/FVC ratio) was 40.1\% greater in the e-cigarette group (95\% Cl 18.2\%-78.4\%, $\mathrm{p}=0.04$ ). Satisfaction with the product was similar in both groups.

Discussion: E-cigarettes are a feasible tool for perioperative smoking cessation in veterans with quit rates comparable to nicotine replacement patch. Spirometry appears to be improved 8-weeks after initiating e-cigarettes compared to nicotine patch, possibly due to worse baseline spirometry and more smoking reduction in the END group. An adequately powered study is recommended to determine if these results can be duplicated. 


\section{TITLE PAGE}

2 1. Title

3 E-cigarettes versus nicotine patch for perioperative smoking cessation: a pilot randomized trial

\section{2. Authors}

5 Susan M. Lee ${ }^{1}$, M.D., F.R.C.P.C., M.A.S.

6 Rachel S. Tenney², B.A.

7 Arthur W. Wallace 2 , M.D., Ph.D.

8 Mehrdad Arjomandi ${ }^{3}$, M.D.

\section{3. Author Affiliations}

10 1.Department of Anesthesia and Perioperative Care, University of California, San Francisco;

11 Anesthesia Service, San Francisco Veterans Affairs Medical Center; Department of

12 Anesthesiology, Pharmacology, and Therapeutics, University of British Columbia; Department

13 of Anesthesia, Royal Columbian Hospital, New Westminster, Canada

14 2. Department of Anesthesia and Perioperative Care, University of California, San Francisco;

15 Anesthesia Service, San Francisco Veterans Affairs Medical Center

$16{ }^{3}$ Department of Pulmonary and Critical Care Medicine, University of California, San Francisco;

17 Department of Pulmonary and Critical Care Medicine, San Francisco Veterans Affairs Medical

18 Center

\section{4. Corresponding Author}

20 Susan M. Lee

21 Department of Anesthesia, Royal Columbian Hospital

22330 East Columbia Street, New Westminster, British Columbia, Canada V3L 3W7

23 Phone: (604) 520-4253 Fax: (604) 224-3400 
$24 \quad$ Email: susanlee.anesthesia@gmail.com, suze.lee@utoronto.ca

25 This work is attributed to the San Francisco Veterans Affairs Medical Center Anesthesiology

26 Service, which is affiliated with the Department of Anesthesia and Perioperative Care,

27 University of California, San Francisco.

28 5. Short Title

29 END Perioperative Smoking Pilot Study 
$30 \underline{\text { Abstract }}$

31 Introduction: Cigarette smoking by surgical patients is associated with increased

32 complications. E-cigarettes have emerged as a potential smoking cessation tool. We sought to

33 determine the feasibility and acceptability of e-cigarettes, compared to nicotine patch, for

34 perioperative smoking cessation in veterans.

35 Methods: Preoperative patients were randomized to either the nicotine patch group $(n=10)$ or the 36 e-cigarette group $(n=20)$. Both groups were given a free 6-week supply in a tapering dose. All 37 patients received brief counseling, a brochure on perioperative smoking cessation, and referral to 38 the California Smokers' Helpline. The primary outcome was rate of smoking cessation on day of surgery confirmed by exhaled carbon monoxide. Secondary outcomes included smoking habits, pulmonary function, adverse events, and satisfaction with the products on day of surgery and at

41 8-weeks follow-up.

42 Results: Biochemically verified smoking cessation on day of surgery was similar in both groups.

43 Change in forced expiratory volume in one second (FEV1) was 592ml greater in the e-cigarette 44 group (95\% CI 153-1031ml, $\mathrm{p}=0.01)$ and change in forced expiratory volume in one second to 45 forced vital capacity ratio (FEV1/FVC ratio) was $40.1 \%$ greater in the e-cigarette group (95\% CI $4618.2 \%-78.4 \%, \mathrm{p}=0.04)$. Satisfaction with the product was similar in both groups.

47 Discussion: E-cigarettes are a feasible tool for perioperative smoking cessation in veterans with 48 quit rates comparable to nicotine replacement patch. Spirometry appears to be improved 849 weeks after initiating e-cigarettes compared to nicotine patch, possibly due to worse baseline 50 spirometry and more smoking reduction in the END group. An adequately powered study is 51 recommended to determine if these results can be duplicated. 


\section{Introduction:}

54 Cigarette smoking is known to increase the risk of surgical site infections, and pulmonary, 55 cardiovascular and other complications in patients undergoing surgery(Moller et al. 2002; Myles 56 et al. 2002; Sorensen \& Jorgensen 2003; Sorensen et al. 2003; Warner 2005). Cigarette smoking

57 is more prevalent in United States veterans than non-veterans, being reported by $25 \%$ of veterans 58 surveyed in 2007, compared to $20 \%$ of non-veteran adults(Brown 2010). Furthermore, veterans 59 may face unique challenges in smoking cessation, given the perception that smoking was a 60 normalized part of military life(Gierisch et al. 2012) and the high rate of coexisting mental health 61 disorders(Duffy et al. 2012). An analysis within the Veterans Affairs (VA) healthcare system 62 showed that pulmonary complications, cardiovascular complications, and surgical site infections 63 were mediators of smoking-associated mortality at 6-months and 1-year after elective 64 surgery(Singh et al. 2013). A systematic review has shown that preoperative smoking cessation 65 therapy improves both short and long-term smoking cessation(Thomsen et al. 2014). In fact, a 66 surgical encounter with the healthcare system has been described as a "teachable moment" that 67 may provide extra motivation for patients to permanently stop smoking. Despite this information, 68 most anesthesiologists do not routinely offer smoking cessation advice to their patients(Kai et al. 69 2008),(Warner et al. 2004).

71 There is currently a desperate need for more data regarding the use of e-cigarettes and their role 72 in smoking cessation interventions(Palazzolo 2013). Despite the fact that the FDA has not 73 approved any e-cigarettes for therapeutic use, e-cigarettes are widely marketed with cessation74 related claims(Grana et al. 2014) and may have the potential to bridge the gap between smoking 75 cigarettes and abstaining. The limited evidence we do have is that e-cigarettes are modestly 
76 effective, similar to transdermal nicotine replacement, in achieving smoking cessation at 6-

77 months with few significant adverse events(Bullen et al. 2013). Several reviews of e-cigarettes

78 have indicated that while e-cigarettes may be helpful for some smokers in quitting smoking, the

79 current evidence is inconclusive due to low quality(Hartmann-Boyce et al. 2016; Malas et al.

80 2016). Despite this paucity of data, a survey of 112 preoperative patients showed that $55 \%$ had

81 tried e-cigarettes and $71 \%$ of those that had tried stated that their reason was to quit smoking. We

82 attempted to determine the acceptability of e-cigarettes as a smoking cessation aid and add to the

83 limited existing data on the safety and efficacy of e-cigarette use in smoking cessation,

84 specifically in the perioperative setting where the risks of continued smoking are great and the

85 motivation to stop is high. The aim of this pilot study was to demonstrate the feasibility of e-

86 cigarettes as a perioperative smoking cessation aid, and to generate preliminary data that might

87 allow for larger studies and improved understanding of the safety and efficacy of e-cigarette use,

88 not only in the perioperative environment, but also more broadly in a public health context. 
90 Methods:

\section{Study design and participants}

92 This was a pilot two arm, parallel group, randomized controlled trial, conducted at the San

93 Francisco Veteran's Affairs Medical Center, which is affiliated with the University of California,

94 San Francisco studying the use of e-cigarettes (versus nicotine patch) for perioperative smoking

95 cessation. Participants were eligible if they presented to the anesthesia preoperative (APO) clinic

96 for elective surgery 3 or more days before surgery, were current cigarette smokers of more than 2

97 cigarettes per day having smoked at least once in the last 7 days, and could provide consent.

98 Participants were excluded if they exclusively used other forms of tobacco (e.g. pipe tobacco) or

99 marijuana only, were pregnant or breast-feeding, had an unstable cardiac condition (e.g. unstable 100 angina, unstable arrhythmia), were currently using smoking cessation pharmacotherapy or were

101 already enrolled in a smoking cessation trial, or currently used e-cigarettes on a daily basis. This 102 study was approved by the UCSF Committee on Human Research (14-15274) and the San

103 Francisco VA Human Research Protection Program and was registered on clinicaltrials.gov

104 (NCT02482233) prior to enrollment of the first patient.

\section{Randomization and Masking}

106 APO patients who met inclusion criteria and provided verbal and written consent were

107 randomized to electronic nicotine devices (END) or nicotine patches (NRT). Randomization was

108 computer-generated, with randomly permuted block sizes of 3 or 6 , in a 2:1 ratio using the ralloc

109 program(Ryan 2011) in Stata version 13 (StataCorp LP, College Station, TX). A 2:1

110 randomization ratio was used because we wanted to gather more experience with feasibility

111 using the newer product (END) in the perioperative population compared to the more thoroughly

112 studied NRT(Lee et al. 2013; Moller et al. 2002; Sorensen et al. 2003; Thomsen et al. 2009). 
113 Allocation was concealed by consecutively numbered, sealed, opaque envelopes. Due to the

114 nature of the intervention, blinding of subjects was not possible. However, healthcare providers

115 were blinded throughout the perioperative period. Outcome adjudicators were blinded wherever

116 possible, but some participants unintentionally unblinded the investigators while reporting side-

117 effects (e.g. reporting a bad taste with inhalation). Smoking cessation and biochemical outcomes

118 were always assessed prior to inquiring about side-effects to minimize this occurrence.

\section{Control Group (NRT)}

120 Patients randomized to the NRT group received a 6-week supply of Nicoderm CQ ${ }^{\circledR}$ patches (5

121 weeks) and placebo patches (1 week) appropriate to baseline nicotine consumption. Those

122 smoking an average of ten or more cigarettes per day were given the $21 \mathrm{mg} /$ day patch for 3

123 weeks, the $14 \mathrm{mg}$ /day patch for 1 week, the $7 \mathrm{mg} /$ day patch for 1 week, and the $0 \mathrm{mg} /$ day patch

124 for 1 week. Participants who reported smoking an average of less than 10 cigarettes per day at

125 baseline were given the $14 \mathrm{mg} /$ day patch for 3 weeks, the $7 \mathrm{mg} /$ day patch for 2 weeks, and the 0

$126 \mathrm{mg} /$ day patch for 1 week. The $0 \mathrm{mg} /$ day patches were clear inert Tegaderm ${ }^{\mathrm{TM}}$ (3M, St. Paul,

$127 \mathrm{MN}$ ) patches cut to the size and shape of the nicotine patch. Participants knew that these patches

128 contained no nicotine. The purpose of the placebo patches was to make the $6^{\text {th }}$ week of the NRT

129 group comparable to the $6^{\text {th }}$ week of the END group, which used no-nicotine e-cigarettes. There

130 is a wide range of NRT products (patch, inhaler, lozenge, gum) commercially available. NRT

131 patches were selected for the control group because prior perioperative smoking cessation studies

132 using NRT have used patches successfully and because they are often first-line therapy in

133 standard clinical use at the VA(Lee et al. 2013; Moller et al. 2002; Sorensen \& Jorgensen 2003;

134 Thomsen et al. 2009). A tapering dose was selected in order to be analogous to the reductions in

135 nicotine concentration in the END group, and because dose-tapering of NRT has been previously 
136 shown to be successful in aiding cessation in both perioperative(Lee et al. 2013) and non-

137 perioperative studies(Hartmann-Boyce et al. 2018; Stead et al. 2012).

138

139 Intervention Group (END)

140 Those allocated to the END group received a 6-week supply of NJOY e-cigarettes (Scottsdale,

141 Arizona, USA) and were instructed to use the Bold (4.5\%) e-cigarettes ad libitum for 3 weeks,

142 the Gold (2.4\%) e-cigarettes ad libitum for 2 weeks and the Study (0\%) e-cigarettes ad libitum

143 for the final week. The number of e-cigarettes issued corresponded to the reported baseline

144 cigarettes smoked per day, calculated assuming 1 NJOY e-cigarette was equivalent to 10

145 cigarettes. The NJOY e-cigarette is a disposable first-generation e-cigarette that is available for

146 purchase in shops and online. The e-cigarette consists of a battery, an atomizer, which heats the

147 solution, and e-liquid (nicotine and propylene glycol). A photo of the study product is shown in

148 Supplementary Figure 1.

149 First generation e-cigarettes were selected at the time of study design, as they were widely

150 available and evidence that second-generation e-cigarettes were more satisfying to smokers was

151 not yet commonly known(Dawkins et al. 2015). By the time the study was approved by the

152 REB, it was felt that changing products would cause significant delays to starting the study.

153 Furthermore, we selected a simple product that did not require charging or e-liquid refills

154 because our population was older with more co-existing disease (e.g. osteoarthritis) than the

155 typical vaping population and wanted to choose the easiest product with the fewest parts.

\section{Study Procedures}

157 Apart from the study product (NRT or END), which was given to patients at the end of the

158 baseline visit, the NRT and END groups underwent identical study procedures. The study 
159 consisted of 3 in-person visits (preoperative baseline, day of surgery, and 8-week follow-up) and

1602 phone-calls (30-day postoperative and 6-month follow-up). At each in-person visit, exhaled

161 carbon monoxide (CO) was measured using the piCO ${ }^{\mathrm{TM}}$ Smokerlyzer ${ }^{\circledR}($ Bedfont Scientific Ltd,

162 Kent, England) and forced expiratory volume in the first second (FEV1) and forced vital

163 capacity (FVC) were measured using the EZOne Spirometer (NDD Medical Technologies, Inc,

164 Andover, MA). A saliva sample was collected for cotinine analysis. At each visit and phone call,

165 smoking status was assessed.

166 Participants in both groups were asked to refrain from the use of cigarettes and all study products

167 at the end of 6-weeks and return unused products at the 8-week visit. Given the lack of long-

168 term safety data for e-cigarettes, we felt it would be unethical to encourage their long-term use.

169 We instructed the NRT group similarly to maintain comparable instructions between groups.

170 Recruitment/Preoperative Baseline Visit:

171 After written informed consent, baseline demographics, smoking habits, exhaled CO, FEV1,

172 FVC and saliva sample were obtained. Participants were educated on the use of both products

173 prior to receiving their allocated product, which was prepared by the study pharmacist and

174 placed in a brown paper bag to mask the contents to the investigator. All participants received

175 brief counseling by the research team, a brochure produced by the American Society of

176 Anesthesiologists explaining the benefits of preoperative smoking cessation, and a referral to the

177 California Smokers' Helpline. The referral was an online form completed by the research team,

178 which would trigger a phone call to the participant by the California Smokers' Helpline.

179 Day of Surgery:

180 Participants were seen by study personnel pre-operatively on their day of surgery. Smoking

181 status, exhaled CO, FEV1, FVC and saliva sample were obtained. Participants were asked about 
182 the occurrence of adverse events or side effects related to use of product. For those whose

183 surgical date was cancelled, a make-up day of surgery visit was scheduled to be as close to the

184 original surgical date as possible.

185 30-Day Postoperative Phone Call:

186 Participants were contacted by phone 30-days post-operatively. If participants could not be

187 reached 30-days post-operatively, subsequent attempts were made until contact was established,

188 to a maximum of 10 attempts. Seven-day point prevalence smoking status was assessed by self-

189 report. Adverse events, side effects, and surgical complications were also assessed.

190 8-Week Post Randomization Visit:

191 Seven-day point prevalence smoking status was assessed and exhaled CO, FEV1, FVC and

192 saliva sample were obtained. Participants were asked about the occurrence of adverse events or

193 side effects related to use of product. After these measures, participants revealed product

194 allocation to study personnel. Study personnel conducted a 30-45 minute long qualitative

195 interview to assess product usage, and participants' attitudes toward both products, the results of

196 which are reported separately. For those that refused an in-person visit, but agreed to telephone

197 interview, exhaled CO, FEV1, FVC and saliva were not obtained. Patients were mailed a $\$ 100$

198 check after completion of the 8-week follow-up visit (or telephone interview).

199 6-month Follow-Up Phone Call:

200 Seven-day point prevalence smoking status and use of e-cigarettes was assessed.

201 Statistical Analysis

202 Power calculation

203 The targeted sample size was 30 (20 intervention, 10 control), balancing cost against precision,

204 as is conventional for a pilot study. Given the small sample size, between-group differences were 
205 not expected to be statistically significant. However, the sample size was intended to provide

206 rough estimates of smoking cessation in each group and provide point estimates and confidence

207 intervals needed for planning a full-scale trial. Regarding participant satisfaction with the

208 product, the sample size did have $80 \%$ power to detect an effect size of 1.09 , accepting an alpha

209 of 0.05 and assuming the standard deviation of a likert scale (0-7) of satisfaction with the

210 intervention (END or NRT) would be around 1.04.

211 Outcome Measures

212 Outcome measures were predefined. The primary outcome was smoking cessation for at least 48

213 hours on the day of surgery, as confirmed by exhaled $\mathrm{CO} \leq 10 \mathrm{ppm}$.

214 Secondary outcomes were:

215 1) Smoking cessation at 30-days postoperatively, 8-week and 6-month follow-up.

216 2) Smoking reduction of $50 \%$ or more (including cessation) compared to, by self-report on

217 the day of surgery, 30-days postoperatively, 8-week and 6-month follow-up.

218 3) Change in FEV1, FVC, and salivary cotinine from baseline to day of surgery and 8-week

219 follow-up.

220 4) Satisfaction with product, description of product as helpful, and recommendation of the

221 product to others, as assessed by agreement on a $0-7$ likert scale on the day of surgery,

222 30-days postoperatively, and 8-week follow-up.

223 5) Adverse events and 30-day postoperative complications.

224 Analysis Plan

225 The study was analyzed by intention-to-treat. No adjustments for multiple comparisons were

226 made, because all outcomes were pre-specified and were limited to a relatively small number. 
227 Descriptive statistics were calculated for baseline demographic variables. Categorical outcomes

228 were analyzed using Fisher exact test. Histograms were constructed for continuous outcomes

229 and visually assessed for distribution and analyzed using Student $t$ test if normally distributed;

230 Wilcoxon rank sum test was used for non-normally distributed variables. A 2-tailed $p$ value of

$231<0.05$ was considered significant. Stata version 13 (StataCorp LP, College Station, TX) was

232 used for all data management and analyses.

233 


\section{Results}

\section{Patient Enrollment}

236 Between August 2015 and February 2016, 30 patients were recruited into the study. Follow-up

237 for the primary outcome was completed in May 2016 and 6-month follow-up calls were

238 completed in August 2016. During recruitment, 1524 patients were booked in the anesthesia

239 preoperative clinic. Of these, $328(21.5 \%)$ were identified as likely smokers based on electronic

240 chart review and 198 were invited to participate in the study. For details, including reasons for

241 missing data, see Consolidated Standards of Reporting Trials (CONSORT) flow chart in Figure

2421.

\section{Baseline Characteristics}

244 Baseline patient characteristics are presented in Table 1. Patient demographics and types of 245 surgery were well balanced. The END group had a higher degree of smoking disease burden, as

246 indicated by a greater number of cigarettes smoked per day, higher Fagerström score for nicotine 247 dependence, increased salivary cotinine and exhaled carbon monoxide levels, and more 248 obstructive spirometry values. Although not statistically significant ( $p=0.37)$, the END group 249 had more diagnosed COPD $(n=6,30 \%)$ than the NRT group $(n=1,10 \%)$ at baseline.

\section{Smoking Cessation Outcomes}

251 Smoking cessation outcomes are presented in Table 2. There were no statistically significant

252 differences between smoking cessation or reduction rates between NRT and END groups at any 253 time point. However, there was a trend towards improved outcomes in the END group at the 8 254 week follow-up visit. Biochemically verified smoking cessation for 2 days preoperatively was 255 achieved in $20 \%(\mathrm{n}=2)$ of the NRT group, which was similar to the $15 \%(\mathrm{n}=3)$ in the END group 256 ( $\mathrm{p}=1.0)$. At 8-week follow-up, no participants in the NRT group had biochemically verified 
257 smoking cessation, while the END group had 3 participants (15\%) that achieved 7-day point-

258 prevalence abstinence $(\mathrm{p}=0.53)$. When including both those that quit and those that reduced

259 cigarette consumption by at least $50 \%, 70 \%$ of the END group $(n=14)$ was able to reduce or quit

260 compared to $40 \%$ of the NRT group $(\mathrm{n}=4)$, but this difference was not statistically significant

261 ( $\mathrm{p}=0.14)$. The number of cigarettes per day smoked by group at baseline, day of surgery, 30-

262 days postoperatively and 8-week follow-up are represented graphically in Supplementary Figure

263 2. Both NRT and END groups reduced their cigarette consumption over time, with median

264 cigarette consumption decreasing from $12.5[\mathrm{IQR}=8-20]$ at baseline to $3[\mathrm{IQR}=0.3-9.5]$ at 8 -

265 week follow-up in the END group ( $\mathrm{p}=0.0001)$ and from $7.8[\mathrm{IQR}=6-20]$ to $5[\mathrm{IQR}=3-8]$ in the

266 NRT group $(\mathrm{p}=0.01)$. There were no statistically significant differences between groups in

267 percentage of smoking reduction at any time point (Table 3).

268 Long-term smoking cessation, as assessed by telephone for 7-day point-prevalence abstinence at

269 6-months, was achieved by $25 \%(\mathrm{n}=5)$ of the END group and $10 \%(\mathrm{n}=1)$ of the NRT group,

270 which was not statistically significantly different $(\mathrm{p}=0.63)$.

271 Spirometry and Cotinine Outcomes

272 Spirometry (FEV1 and FEV1/FVC ratios), salivary cotinine, and exhaled CO were analyzed by

273 comparing changes from baseline between NRT and END groups and are presented in Table 3.

274 On the day of surgery, both NRT and END groups experienced reductions in FEV1 and

275 FEV1/FVC ratios of similar magnitude and were not statistically significantly different $(\mathrm{p}=0.75$

276 and $\mathrm{p}=0.09$ respectively). At 8 -weeks after randomization, the END group had greater

277 improvement in FEV1, FEV1/FVC ratio, with increases of FEV1 of 292ml (SD 503ml) and

278 FEV1/FVC ratio of 2\% (SD 10.5\%), compared to the NRT group, which experienced a decrease

279 in FEV1 of 300ml (SD 497ml) and a reduction in FEV1/FVC ratio of 38\% (SD 79\%). These 
280 differences were statistically significant for change in FEV1 $(p=0.01)$ and for change in

281 FEV1/FVC ratio $(\mathrm{p}=0.04)$. Point estimates for cotinine and exhaled CO reductions were greater

282 in the END group on both the day of surgery and 8-week follow-up visits, but no differences

283 were statistically significant between NRT and END groups.

\section{Adverse Events and Postoperative Complications}

285 No participants in either group experienced severe adverse events at any time point. As shown 286 in Table 4, adverse event rates were similar between groups on the day of surgery ( $50 \%$ in the 287 NRT group experienced at least one adverse event compared to 53\% in the END group, $\mathrm{p}=1.0$ ) 288 and at 8 -week follow-up (33\% in the NRT group versus 50\% in the END group, $\mathrm{p}=0.45$ ).

289 Product usage was similar between groups (Table 5).

290 No participants in either group experienced intraoperative complications. The rate of 291 postoperative complications was similar in both groups $(60 \%$ in the NRT group and $26 \%$ in the 292 END group, $\mathrm{p}=0.11)$.

293 Common adverse events related to both NRT and END included headache, nausea, cough, and

294 throat irritation, as shown in Table 6. There were no statistically significant differences between 295 event rates in each group.

\section{Smokers' Helpline Usage}

297 The California Smokers' Helpline indicated that contact was made with just over half of the 298 participants. Use of services was similar in the END and NRT groups, as shown in Table 7.

\section{Satisfaction Outcomes}

300 The acceptability of nicotine patches and e-cigarettes were assessed on the day of surgery and 8301 weeks after randomization. Regular (daily or most days) use of the product was not statistically 302 significantly different between NRT and END groups $(\mathrm{p}=0.71)$, with about half reporting regular 
303 usage on the day of surgery. Usage increased by 8-week follow-up, particularly in the END

304 group, which reported $80 \%(\mathrm{n}=16)$ regular use compared with $67 \%(\mathrm{n}=6)$ in the NRT group,

305 although the difference was not statistically significant $(\mathrm{p}=0.64)$.

306 Satisfaction was also similar at both time points between NRT and END groups, with both

307 groups being at least somewhat satisfied with the products they were given, as shown in Table 5.

308 More in-depth explorations of how each product was used, patient attitudes towards smoking

309 cessation in relation to the assigned products, and satisfaction with the products were conducted

310 in qualitative interviews at 8-weeks follow-up and will be reported separately.

\section{Feasibility of Study Processes}

312 As a pilot study, feasibility of study processes were also analyzed. As with prior perioperative

313 smoking cessation studies(Lee et al. 2013), potential research participants were successfully

314 identified by chart review in the preoperative period, although similarly, more than half of

315 smokers chose not to participate in the research study despite meeting eligibility criteria (Figure

316 1). Recruitment may have been further reduced in this trial compared to prior perioperative

317 smoking cessation studies due to the use of non-standard treatments (ENDs) compared to other

318 perioperative studies that used NRT or varenicline(Moller et al. 2002; Thomsen et al. 2009;

319 Wong et al. 2017). Blinding of research staff was successful, although it was not feasible to have

320 participants blinded given the nature of the treatment. Follow-up was nearly complete, with

321 losses to follow-up for the primary outcome occurring only due to cancelled surgeries. Future

322 studies may wish to select a fixed date follow-up (such as 8-weeks post-randomization) for the

323 primary outcome, regardless of surgical date, to maintain consistency between participants and

324 reduce losses due to cancelled and postponed surgeries. 


\section{Discussion}

326 In this pilot randomized trial of END versus NRT initiated in the preoperative period, we found

327 that e-cigarettes were a feasible and acceptable intervention to veterans around the time of

328 surgery and had similar smoking cessation rates compared to transdermal nicotine replacement.

329 Given the current need for more controlled data with respect to the use of e-cigarettes for

330 smoking cessation(Hartmann-Boyce et al. 2016) (a practice already popular amongst

331 preoperative patients(Kadimpati et al. 2015)), this trial has demonstrated important groundwork

332 for future studies.

333 Our findings are consistent with those of the largest randomized controlled trial of e-cigarettes

334 versus NRT to date(Bullen et al. 2013) and several recent systematic reviews(Hartmann-Boyce

335 et al. 2016; Malas et al. 2016), which also found that e-cigarettes were modestly effective in

336 helping smokers quit compared to NRT. Our protocol differed in its shorter duration of therapy

337 (6-weeks) and tapering nicotine dose in both NRT and END groups, suggesting that a shorter

338 duration of therapy may still be effective in assisting patients in quitting smoking. Similar to

339 prior e-cigarette studies(Hartmann-Boyce et al. 2016), none of our participants experienced

340 serious adverse events, although mild and moderate headache, nausea, cough and throat irritation

341 were common. Quit rates in this study (30\% in NRT group and 25\% in the END group at 30 -

342 days postoperatively) were similar to those found in another pragmatic perioperative smoking

343 cessation study using NRT(Lee et al. 2013) (quit rate at 30-days postoperatively in the NRT

344 group was 29\% versus usual care 11\%), indicating that both e-cigarettes and NRT are probably

345 more effective than no intervention and can be useful tools to assist patients in quitting smoking

346 at a time when they are highly motivated to quit due to impending or recent surgery. 
347 The differential in exposure time to END versus NRT products prior to the day of surgery

348 (average of 16 versus 11 days) may have introduced some difficulty in interpreting the primary

349 outcome. Future studies may choose a fixed time interval post-randomization (similar to our 8-

350 week follow-up visit) rather than the day of surgery as a primary outcome to standardize this

351 variable between groups.

352 The improvements in spirometry, for example the increased FEV1 and FEV1/FVC in the END

353 group may indicate that e-cigarette vapor is less harmful than continued cigarette smoking, a

354 claim that is often marketed(Grana \& Ling 2014) despite some controversy in the

355 literature(Konstantinos \& Riccardo 2014; Palazzolo 2013). These results are especially

356 impressive in light of the fact that baseline characteristics show that the END group was

357 imbalanced towards heavier, more dependent smokers with worse baseline spirometry values.

358 The results could also be explained by a higher baseline prevalence of COPD in the END group

359 in addition to the possible increased smoking reduction and cessation in the END group. Given

360 the small sample size and effort-dependence of spirometry, further studies would be needed to

361 verify the consistency of the spirometry findings.

362 As is typical of pilot studies, our study was limited by small sample size, such that we were

363 underpowered to detect all but the largest of differences between groups. Nevertheless, we were

364 able to demonstrate the acceptability of e-cigarettes for perioperative smoking cessation, as

365 evidenced by similar satisfaction scores between END and NRT groups, with most patients in

366 the END group indicating that the product was helpful in their quit attempt and that they would

367 recommend the product.

368 Participants were given a set 6-week supply based on baseline cigarettes per day of either END

369 (per manufacturer's recommendation) or NRT (daily use) upon enrollment to the study. NRT 
370 patients were directed to use the patches daily, while END patients were asked to use the e-

371 cigarette devices ad libitum, with the further instruction that if they ran out of a particular

372 strength of product, they should move on to the next product. A limitation to the study may be

373 that the set amount of product given to the END group may not have been truly ad libitum due to

374 the limited supply. Nevertheless, most participants (15/20 (75\%) of the END group) had leftover

375 e-cigarettes and therefore did achieve true ad libitum use.

376 Another limitation to this study was the inconsistent use of behavioral support. While most

377 referrals were appropriately received by the California Smokers' Helpline, a large proportion

378 were unable to connect with counselling support. It is likely that with better adherence to

379 telephone counselling, quit rates in both groups may have been higher. Future studies may

380 benefit from tighter control of access and use of smoking cessation counselling, including face-

381 to-face counselling and improved follow-up counselling, which might improve smoking

382 cessation rates in both arms, as intensive smoking cessation counselling has previously been

383 shown to be effective in the perioperative period(Thomsen et al. 2014).

384

385 Conclusions

386 E-cigarettes were found to be a feasible and acceptable aid for perioperative smoking cessation

387 with quit rates comparable to nicotine replacement patch. Spirometry may be improved with e-

388 cigarette use. A large adequately powered study is recommended to determine if the results from 389 this pilot study can be duplicated. 


\section{Acknowledgements}

392 The authors wish to acknowledge research assistants Sarah Dalton and Wendy Chin from the

393 Arjomandi Lab and Rosalind Franklin University medical student Christopher Sirivoranankul for

394 their assistance in carrying out this study. The cooperation of the Benowitz Lab is also

395 appreciated for performing salivary cotinine analyses. 
$\underline{\text { References }}$

397

398

399

400

401

402

403

404

405

406

407

408

409

410

411

412

413

414

415

416

417

418

419

420

421

422

423

424

425

426

427

428

429

430

431

432

433

434

435

436

437

438

Brown DW. 2010. Smoking prevalence among US veterans. J Gen Intern Med 25:147-149. 10.1007/s11606-009-1160-0

Bullen C, Howe C, Laugesen M, McRobbie H, Parag V, Williman J, and Walker N. 2013. Electronic cigarettes for smoking cessation: a randomised controlled trial. Lancet 382:1629-1637. 10.1016/s0140-6736(13)61842-5

Dawkins L, Kimber C, Puwanesarasa Y, and Soar K. 2015. First- versus second-generation electronic cigarettes: predictors of choice and effects on urge to smoke and withdrawal symptoms. Addiction 110:669-677. 10.1111/add.12807

Duffy SA, Kilbourne AM, Austin KL, Dalack GW, Woltmann EM, Waxmonsky J, and Noonan D. 2012. Risk of smoking and receipt of cessation services among veterans with mental disorders. Psychiatr Serv 63:325-332. 10.1176/appi.ps.201100097

Gierisch JM, Straits-Troster K, Calhoun PS, Beckham JC, Acheson S, and Hamlett-Berry K. 2012. Tobacco use among Iraq- and Afghanistan-era veterans: a qualitative study of barriers, facilitators, and treatment preferences. Prev Chronic Dis 9:E58.

Grana R, Benowitz N, and Glantz SA. 2014. E-Cigarettes A Scientific Review. Circulation 129:1972-1986. 10.1161/circulationaha.114.007667

Grana RA, and Ling PM. 2014. "Smoking revolution": a content analysis of electronic cigarette retail websites. Am J Prev Med 46:395-403. 10.1016/j.amepre.2013.12.010

Hartmann-Boyce J, Chepkin SC, Ye W, Bullen C, and Lancaster T. 2018. Nicotine replacement therapy versus control for smoking cessation. Cochrane Database Syst Rev 5:CD000146. 10.1002/14651858.CD000146.pub5

Hartmann-Boyce J, McRobbie H, Bullen C, Begh R, Stead LF, and Hajek P. 2016. Electronic cigarettes for smoking cessation. Cochrane Database Syst Rev 9:CD010216. 10.1002/14651858.CD010216.pub3

Kadimpati S, Nolan M, and Warner DO. 2015. Attitudes, beliefs, and practices regarding electronic nicotine delivery systems in patients scheduled for elective surgery. Mayo Clin Proc 90:71-76. 10.1016/j.mayocp.2014.11.005

Kai T, Maki T, Takahashi S, and Warner DO. 2008. Perioperative tobacco use interventions in Japan: a survey of thoracic surgeons and anaesthesiologists. Br J Anaesth 100:404410. aem400 [pii]

$10.1093 / \mathrm{bja} / \mathrm{aem} 400$ [doi]

Konstantinos EF, and Riccardo P. 2014. Safety evaluation and risk assessment of electronic cigarettes as tobacco cigarette substitutes: a systematic review. Ther Adv Drug Saf 5:67-86. 10.1177/2042098614524430

Lee SM, Landry J, Jones PM, Buhrmann 0, and Morley-Forster P. 2013. The effectiveness of a perioperative smoking cessation program: a randomized clinical trial. Anesth Analg 117:605-613. 10.1213/ANE.0b013e318298a6b0

Malas M, van der Tempel J, Schwartz R, Minichiello A, Lightfoot C, Noormohamed A, Andrews J, Zawertailo L, and Ferrence R. 2016. Electronic Cigarettes for Smoking Cessation: A Systematic Review. Nicotine Tob Res 18:1926-1936. $10.1093 /$ ntr/ntw119 
439

440

441

442

443

444

445

446

447

448

449

450

451

452

453

454

455

456

457

458

459

460

461

462

463

464

465

466

467

468

469

470

471

472

473

474

475

476

477

478

479

480

481

482

483

484

Moller AM, Villebro N, Pedersen T, and Tonnesen H. 2002. Effect of preoperative smoking intervention on postoperative complications: a randomised clinical trial. Lancet 359:114-117. S0140-6736(02)07369-5 [pii]

10.1016/S0140-6736(02)07369-5 [doi]

Myles PS, Iacono GA, Hunt JO, Fletcher H, Morris J, McIlroy D, and Fritschi L. 2002. Risk of respiratory complications and wound infection in patients undergoing ambulatory surgery: smokers versus nonsmokers. Anesthesiology 97:842-847. 00000542200210000-00015 [pii]

Palazzolo DL. 2013. Electronic cigarettes and vaping: a new challenge in clinical medicine and public health. A literature review. Front Public Health 1:56. 10.3389/fpubh.2013.00056

Ryan P. 2011. RALLOC: Allocation of treatments in controlled trials using random permuted blocks.

Singh JA, Hawn M, Campagna EJ, Henderson WG, Richman J, and Houston TK. 2013. Mediation of smoking-associated postoperative mortality by perioperative complications in veterans undergoing elective surgery: data from Veterans Affairs Surgical Quality Improvement Program (VASQIP)--a cohort study. BMJ Open 3. 10.1136/bmjopen-2012-002157

Sorensen LT, and Jorgensen T. 2003. Short-term pre-operative smoking cessation intervention does not affect postoperative complications in colorectal surgery: a randomized clinical trial. Colorectal Dis 5:347-352. 450 [pii]

Sorensen LT, Karlsmark T, and Gottrup F. 2003. Abstinence from smoking reduces incisional wound infection: a randomized controlled trial. Ann Surg 238:1-5. 10.1097/01.SLA.0000074980.39700.31 [doi]

00000658-200307000-00001 [pii]

Stead LF, Perera R, Bullen C, Mant D, Hartmann-Boyce J, Cahill K, and Lancaster T. 2012. Nicotine replacement therapy for smoking cessation. Cochrane Database of Systematic Reviews. 10.1002/14651858.CD000146.pub4

Thomsen T, Tonnesen H, and Moller AM. 2009. Effect of preoperative smoking cessation interventions on postoperative complications and smoking cessation. Br J Surg 96:451-461. 10.1002/bjs.6591 [doi]

Thomsen T, Villebro N, and Moller AM. 2014. Interventions for preoperative smoking cessation. Cochrane Database Syst Rev 3:Cd002294.

10.1002/14651858.CD002294.pub4

Warner DO. 2005. Helping surgical patients quit smoking: why, when, and how. Anesth Analg 101:481-487. 101/2/481 [pii]

10.1213/01.ANE.0000159152.31129.84 [doi]

Warner DO, Sarr MG, Offord KP, and Dale LC. 2004. Anesthesiologists, general surgeons, and tobacco interventions in the perioperative period. Anesth Analg 99:1766-1773. 99/6/1766 [pii]

10.1213/01.ANE.0000136773.40216.87 [doi]

Wong J, Abrishami A, Riazi S, Siddiqui N, You-Ten E, Korman J, Islam S, Chen X, Andrawes MS, Selby P, Wong DT, and Chung F. 2017. A Perioperative Smoking Cessation Intervention With Varenicline, Counseling, and Fax Referral to a Telephone Quitline Versus a Brief Intervention: A Randomized Controlled Trial. Anesth Analg. 10.1213/ANE.0000000000001894 
485

486

487

PeerJ reviewing PDF | (2018:02:25904:1:1:NEW 30 Jul 2018) 


\section{Figure 1 (on next page)}

Consolidated Standards of Reporting Trials flow chart indicating recruitment, randomization and retention of trial participants.

Of the 35 patients approached for inclusion but found to be ineligible, the reasons for ineligibility included: smoking less than 2 cigarettes per day $(n=10)$, already being on smoking cessation pharmacotherapy $(n=9)$, smoking non-cigarette tobacco only $(n=5)$, prior adverse reaction to NRT patch $(n=3)$, already enrolled in smoking cessation program $(n=3)$, regular use of e-cigarettes $(n=2)$, surgical date changed $(n=2)$ and currently experiencing an unstable cardiac condition $(n=1)$. One patient was found to be ineligible after consent, but prior to randomization. All patients were given the treatment (END or NRT) to which they were randomized. Losses to follow-up were minimal and balanced between groups. 
Preoperative patients presenting to clinic at least 3days before surgery $(n=1524)$

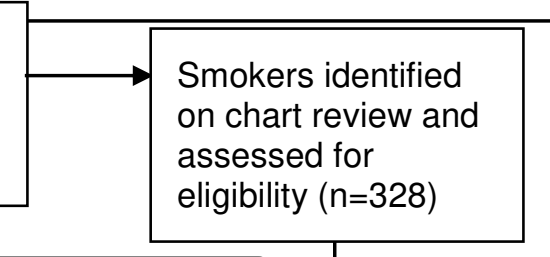

Enrollment
Smokers identified on chart review and assessed for eligibility $(n=328)$
Lost to follow-up $(n=1)$

- Surgery cancelled after pre-op data collected $(n=1)$
Allocated to control (NRT) group $(n=10)$

- Received nicotine patches $(n=10)$
Non-smokers $(n=1196)$

Excluded $(\mathrm{n}=298)$

- Not actually a current smoker $(n=47)$

- Ineligible $(n=35)$

- Declined to participate $(n=86)$

- Not assessed due to scheduling problems $(\mathrm{n}=80)$

- Did not show up to clinic $(n=50)$
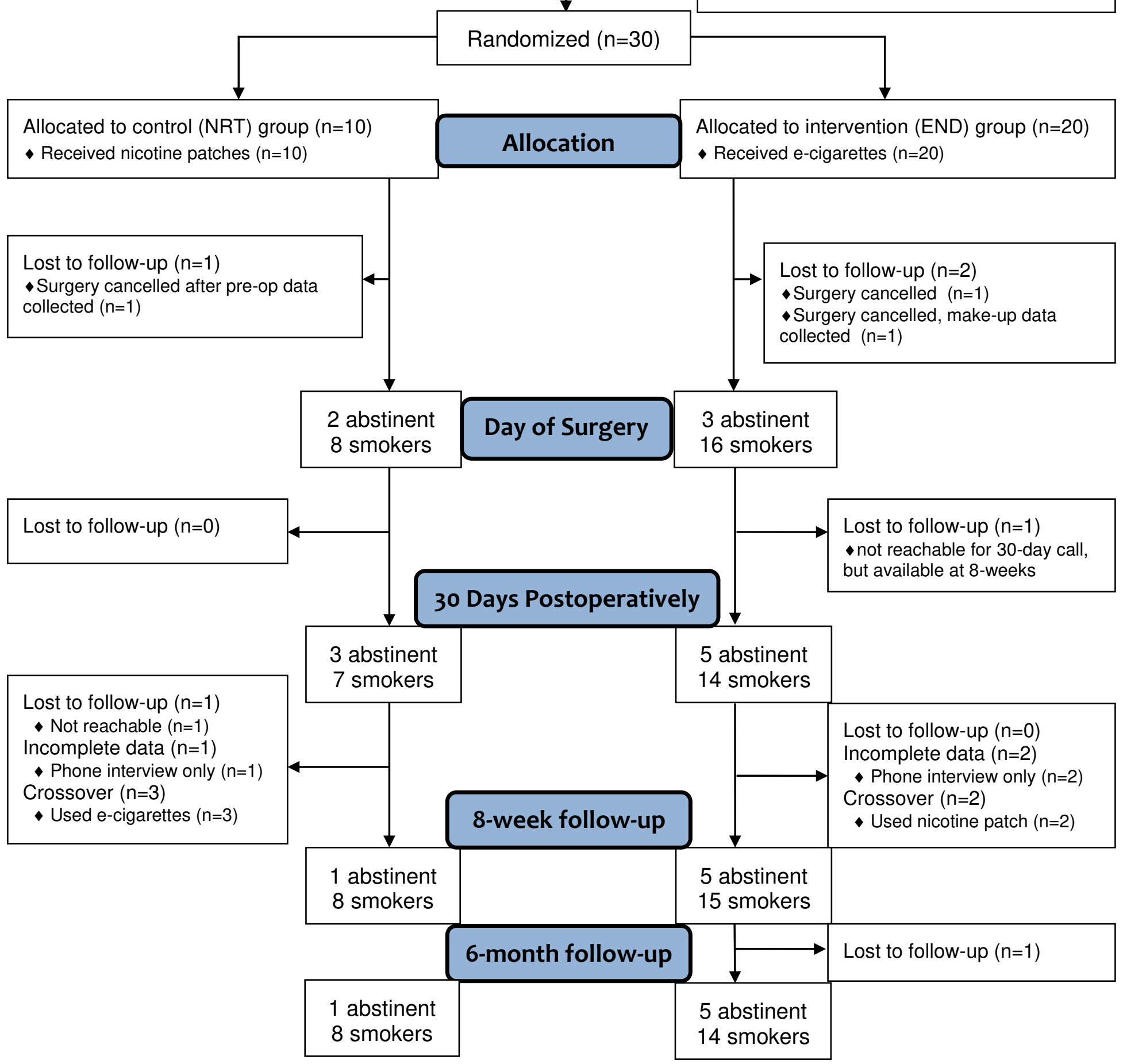


\section{Table $\mathbf{1}$ (on next page)}

Baseline characteristics

Values are mean (SD) or $n$ (percentage). Percentages may not add to 100 due to rounding. $\mathrm{NRT}=$ nicotine replacement therapy. END = electronic nicotine delivery (e-cigarette). $\mathrm{BMI}=$ body mass index $=\left(\right.$ weight $[\mathrm{kg}] /$ height $\left.^{2}\left[\mathrm{~m}^{2}\right]\right)$; COPD $=$ chronic obstructive pulmonary disease; $\mathrm{CO}$ = carbon monoxide; FEV1 = forced expiratory volume in first second; FVC = forced vital capacity *Other surgery includes ophthalmology, urology, otolaryngology, plastics, gynecology and podiatry ${ }^{\dagger}$ Heart disease defined as coronary artery disease, congestive heart failure, or arrhythmia 
Table 1. Baseline Characteristics

\begin{tabular}{|c|c|c|}
\hline Characteristic & NRT Group $(n=10)$ & END Group $(n=20)$ \\
\hline \multicolumn{3}{|l|}{ Demographics } \\
\hline Male & $9(90.0 \%)$ & $18(90.0 \%)$ \\
\hline Age (years) & $53(10.6)$ & $54(12.7)$ \\
\hline Height (cm) & $179.8(8.9)$ & $180.7(7.7)$ \\
\hline Weight (kg) & $92(25.9)$ & $97(19.7)$ \\
\hline Body mass index $\left(\mathrm{kg} / \mathrm{m}^{2}\right)$ & 28.5 (7.9) & $29.6(5.8)$ \\
\hline Race (white) & $5(50.0 \%)$ & $11(55.0 \%)$ \\
\hline Ethnicity (latino) & $0(0 \%)$ & $2(10.0 \%)$ \\
\hline \multicolumn{3}{|l|}{ Surgery Details } \\
\hline General surgery & $3(30.0 \%)$ & $5(25.0 \%)$ \\
\hline Orthopedic surgery & $2(20.0 \%)$ & $6(30.0 \%)$ \\
\hline Neurosurgery & $1(10.0 \%)$ & $1(5.0 \%)$ \\
\hline Vascular surgery & $1(5.0 \%)$ & $0(0 \%)$ \\
\hline Other surgery type* & $4(40.0 \%)$ & $7(35.0 \%)$ \\
\hline Ambulatory surgery & $7(70.0 \%)$ & $13(65.0 \%)$ \\
\hline $\begin{array}{l}\text { Days seen prior to } \\
\text { scheduled surgery }\end{array}$ & $16.5(9.5)$ & 11.2 (7.9) \\
\hline \multicolumn{3}{|l|}{ Comorbidities } \\
\hline Diabetes & $0(0 \%)$ & $2(10.0 \%)$ \\
\hline Hypertension & $3(30.0 \%)$ & $7(35.0 \%)$ \\
\hline Heart disease $^{+}$ & $0(0 \%)$ & $1(5.0 \%)$ \\
\hline COPD & $1(10.0 \%)$ & $6(30.0 \%)$ \\
\hline \multicolumn{3}{|l|}{ Smoking Habits } \\
\hline Cigarettes smoked per day & $10.8(6.6)$ & $15.3(10.5)$ \\
\hline Number of years smoking & $32(16.4)$ & $32(15.6)$ \\
\hline Pack-years smoking history & $16.7(12.1)$ & $26.4(27.0)$ \\
\hline $\begin{array}{l}\text { Fagerström score (out of } \\
10 \text { ) }\end{array}$ & $2.5(0.85)$ & $3.7(2.6)$ \\
\hline \multicolumn{3}{|l|}{ Laboratory indices } \\
\hline Salivary cotinine (ng/ml) & $130.1(75.3)$ & $209.6(110.3)$ \\
\hline Exhaled CO level (ppm) & $16.1(7.7)$ & $21.7(11.5)$ \\
\hline FEV1 (L) & $3.14(1.35)$ & $2.78(1.11)$ \\
\hline FVC (L) & $3.52(1.28)$ & $4.03(1.32)$ \\
\hline FEV1/FVC ratio (\%) & $105 \%(81.3 \%)$ & $68.2 \%(13.0 \%)$ \\
\hline
\end{tabular}

1 Values are mean (SD) or $n$ (percentage). Percentages may not add to 100 due to rounding. NRT

2 = nicotine replacement therapy. END = electronic nicotine delivery (e-cigarette). 
$3 \mathrm{BMI}=$ body mass index $=\left(\right.$ weight $[\mathrm{kg}] /$ height $\left.^{2}\left[\mathrm{~m}^{2}\right]\right) ; \mathrm{COPD}=$ chronic obstructive pulmonary

4 disease; $\mathrm{CO}=$ carbon monoxide; FEV1 = forced expiratory volume in first second; $F V C=$ forced

5 vital capacity

6 *Other surgery includes ophthalmology, urology, otolaryngology, plastics, gynecology and

7 podiatry

$8{ }^{\dagger}$ Heart disease defined as coronary artery disease, congestive heart failure, or arrhythmia 
Table 2 (on next page)

Smoking cessation outcomes 
1

Table 2. Smoking cessation outcomes

\begin{tabular}{|c|c|c|c|c|}
\hline Outcome & $\begin{array}{l}\text { NRT Group } \\
(n=10)\end{array}$ & $\begin{array}{l}\text { END Group } \\
(n=20)\end{array}$ & $\begin{array}{c}\text { Relative Risk } \\
(95 \% \mathrm{Cl}) \\
\end{array}$ & $p$ \\
\hline \multicolumn{5}{|l|}{ Day of surgery } \\
\hline Smoking cessation (verified*) & $2(20 \%)$ & $3(15 \%)$ & $0.75(0.15-3.79)$ & 1.0 \\
\hline $\begin{array}{l}\text { Smoking cessation (self- } \\
\text { report) }\end{array}$ & $3(30 \%)$ & $4(20 \%)$ & $0.67(0.18-2.42)$ & 0.66 \\
\hline $\begin{array}{l}\text { Smoking reduction (including } \\
\text { cessation) }{ }^{\dagger}\end{array}$ & $7(70 \%)$ & $13(65 \%)$ & $0.93(0.55-1.56)$ & 1.0 \\
\hline \multicolumn{5}{|l|}{ 30-days postoperatively } \\
\hline $\begin{array}{l}\text { Smoking cessation (self- } \\
\text { report) }\end{array}$ & $3(30 \%)$ & $5(25 \%)$ & $0.83(0.25-2.80)$ & 1.0 \\
\hline $\begin{array}{l}\text { Smoking reduction (including } \\
\text { cessation) }{ }^{\dagger}\end{array}$ & $5(50 \%)$ & $9(45 \%)$ & $0.90(0.41-1.98)$ & 1.0 \\
\hline \multicolumn{5}{|l|}{ 8-weeks after randomization } \\
\hline Smoking cessation (verified ${ }^{*}$ ) & $0(0 \%)$ & $3(15 \%)$ & RR=undefined $\neq$ & 0.53 \\
\hline $\begin{array}{l}\text { Smoking cessation (self- } \\
\text { report) }\end{array}$ & $1(10 \%)$ & $5(25 \%)$ & $2.5(0.34-18.6)$ & 0.63 \\
\hline $\begin{array}{l}\text { Smoking reduction (including } \\
\text { cessation) }{ }^{\dagger}\end{array}$ & $4(40 \%)$ & $14(70 \%)$ & $1.75(0.78-3.94)$ & 0.14 \\
\hline \multicolumn{5}{|l|}{ 6-month follow-up } \\
\hline $\begin{array}{l}\text { Smoking cessation (self- } \\
\text { report) }\end{array}$ & $1(10 \%)$ & $5(25 \%)$ & $2.5(0.34-18.6)$ & 0.63 \\
\hline $\begin{array}{l}\text { Smoking reduction (including } \\
\text { cessation) }{ }^{\dagger}\end{array}$ & $5(50 \%)$ & $6(30 \%)$ & $0.62(0.31-1.24)$ & 0.43 \\
\hline
\end{tabular}

2 Values are $n$ (percentage). p-values from Fisher's exact test. Relative risks were END

3 versus NRT. NRT = nicotine replacement therapy. END = electronic nicotine delivery $(\mathrm{e}-$

4 cigarette). Cessation on the day of surgery was determined based on 48-hour point

5 prevalence abstinence. Cessation at all other time points was determined by 7-day point-

6 prevalence abstinence. Smoking reduction includes those that quit.

$7 \quad$ *Smoking cessation verified by exhaled carbon monoxide $10 \mathrm{ppm}$ or less.

$8{ }^{\dagger}$ Smoking reduction is defined by reduction of $50 \%$ or more cigarettes per day compared to

9 baseline, including smoking cessation. 
10 Analysis by intention-to-treat - those lost to follow-up were assumed to have continued

11 smoking.

$12 \quad$ ₹Relative risk undefined due to no quitters in the NRT group, risk difference $=15 \%(95 \% \mathrm{Cl}$ -

$136.5 \%$ to $+30.6 \%)$

14 
Table 3 (on next page)

Laboratory outcomes 


\begin{tabular}{|c|c|c|c|c|}
\hline Outcome & NRT Group & END Group & $\begin{array}{c}\text { Difference } \\
\text { (95\% Cl of difference) }\end{array}$ & $p$ \\
\hline Day of surgery & $(n=10)$ & $\left(n=18^{*}\right)$ & & \\
\hline FEV1 (ml) change & $-236(585)$ & $-163(549)$ & $73(-383$ to +528$)$ & 0.75 \\
\hline FEV1/FVC ratio (\%) change & $-32.2 \%(74 \%)$ & $-1.6 \%(8.2 \%)$ & $\begin{array}{c}+30.6 \%(-5.3 \% \text { to } \\
+66.5 \%)\end{array}$ & 0.09 \\
\hline Cotinine $(\mathrm{ng} / \mathrm{ml})$ change & +106 (137) & +19 (119) & $-87(-189$ to +14$)$ & 0.09 \\
\hline Exhaled CO (ppm) change & $+1.9(7.2)$ & $-1.7(10.7)$ & $-3.6(-11.4$ to +4.2$)$ & 0.35 \\
\hline $\begin{array}{l}\text { Percentage reduction of } \\
\text { cigarettes smoked per day }\end{array}$ & $49 \%(45 \%)$ & $59 \%(37 \%)$ & $10 \%$ (42\% to $-22 \%)$ & 0.52 \\
\hline \multicolumn{5}{|l|}{ 30-days postoperatively } \\
\hline $\begin{array}{l}\text { Percentage reduction of } \\
\text { cigarettes smoked per day }\end{array}$ & $51 \%(31 \%)$ & $33 \%(49 \%)$ & $-18 \%(22 \%$ to $-61 \%)$ & 0.39 \\
\hline 8-weeks after randomization & $(n=8)$ & $(n=18)$ & & \\
\hline FEV1 (ml) change & $-300(497)$ & $+292(503)$ & $+592(+153$ to +1032$)$ & 0.01 \\
\hline FEV1/FVC ratio (\%) change & $\begin{array}{l}-38.1 \% \\
(79.2 \%)\end{array}$ & $+2.0 \%(10.5 \%)$ & $\begin{array}{c}+40.1 \%(+1.8 \% \text { to } \\
+78.4 \%)\end{array}$ & 0.04 \\
\hline Cotinine $(\mathrm{ng} / \mathrm{ml})$ change & +34 (89) & $-48(103)$ & $-82(-169$ to +5$)$ & 0.06 \\
\hline Exhaled CO (ppm) change & $+7.1(11.0)$ & $-2.1(12.2)$ & $-9.2(-19.6$ to +1.2$)$ & 0.08 \\
\hline $\begin{array}{l}\text { Percentage reduction of } \\
\text { cigarettes smoked per day }\end{array}$ & $47 \%(41 \%)$ & $64 \%(31 \%)$ & $17 \%$ (45\% to $-12 \%)$ & 0.23 \\
\hline \multicolumn{5}{|l|}{6 months after randomization } \\
\hline $\begin{array}{l}\text { Percentage reduction of } \\
\text { cigarettes smoked per day }\end{array}$ & $56 \%(31 \%)$ & $33 \%(35 \%)$ & $-23 \%$ (8\% to $-55 \%)$ & 0.14 \\
\hline
\end{tabular}

1 All values are reported as changes compared to baseline.

$2 \mathrm{CO}$ = carbon monoxide; FEV1 = forced expiratory volume in first second; FVC = forced vital

3 capacity

4 Values are mean (standard deviation). p-values from two-sided t-tests.

5 Percentage reduction of cigarettes smoked per day refers to the reduction in smoking

6 compared to baseline, where cessation would be $100 \%$ reduction and reducing from 20

7 cigarettes per day to 10 cigarettes per day would be a $50 \%$ reduction. 
$8{ }^{*} \mathrm{n}=18$ for day of surgery FEV1, FEV1/FVC, and cotinine, but $\mathrm{n}=19$ for exhaled CO because one

9 patient in the END group agreed to do exhaled CO, but refused all other tests.

10

11 


\section{Table 4(on next page)}

Adverse Events and Postoperative complications

Values are $n$ (percentage). p-values from Fisher's exact test. Severity of adverse events classified as mild if self-limited and no intervention required, moderate if it required intervention (e.g. oral analgesic for headache), and severe if it required hospitalization. There were no severe adverse events reported in either group at any time point. No participants experienced intraoperative complications. "Two participants in the END group experienced PACU complications (non-cardiac chest pain, which resolved in PACU and wheezing, which resolved with albuterol administered in PACU). 'Two participants in the NRT group experienced self-reported postoperative complications within 30-days (both wound complications), while 5 participants in the END group had postoperative complications (2 wound-related, 2 bladder-related and 1 respiratory). ${ }^{\ddagger}$ All complications assessed by chartreview were wound-related in both NRT and END groups. NRT = nicotine replacement therapy. END = electronic nicotine delivery (e-cigarette). PACU = post-anesthesia care unit. 
1

\begin{tabular}{|c|c|c|c|}
\hline Outcome & NRT Group & END Group & $p$ \\
\hline Day of surgery & $(n=10)$ & $(n=19)$ & \\
\hline $\begin{array}{l}\text { Number with any adverse } \\
\text { event, } \mathrm{n}(\%)\end{array}$ & $5(50 \%)$ & $10(53 \%)$ & 1.0 \\
\hline $\begin{array}{l}\text { Number with moderate } \\
\text { adverse event, } \mathrm{n}(\%)\end{array}$ & $0(0 \%)$ & $1(5 \%)$ & 1.0 \\
\hline PACU complications* ${ }^{*}$ & $0(0 \%)$ & $2(11 \%)$ & 0.53 \\
\hline 30-days postoperatively & $(n=10)$ & $(n=19)$ & \\
\hline $\begin{array}{l}\text { Number with any adverse } \\
\text { event }\end{array}$ & $5(50 \%)$ & $7(37 \%)$ & 0.69 \\
\hline $\begin{array}{l}\text { Number with moderate } \\
\text { adverse event, } \mathrm{n}(\%)\end{array}$ & $0(0 \%)$ & $1(5 \%)$ & 1.0 \\
\hline $\begin{array}{l}\text { Postoperative complications } \\
\text { (by self-report) }{ }^{\dagger}\end{array}$ & $2(20 \%)$ & $5(26 \%)$ & 1.0 \\
\hline $\begin{array}{l}\text { Postoperative complications } \\
\text { (by chart review })^{\ddagger}\end{array}$ & $6(60 \%)$ & $5(26 \%)$ & 0.11 \\
\hline 8-weeks after randomization & $(n=9)$ & $(n=20)$ & \\
\hline $\begin{array}{l}\text { Number with any adverse } \\
\text { event }\end{array}$ & $3(33 \%)$ & $10(50 \%)$ & 0.45 \\
\hline $\begin{array}{l}\text { Number with moderate } \\
\text { adverse events, } \mathrm{n}(\%)\end{array}$ & $1(11 \%)$ & $1(5 \%)$ & 0.53 \\
\hline
\end{tabular}

2 Values are $n$ (percentage). p-values from Fisher's exact test.

3 Severity of adverse events classified as mild if self-limited and no intervention required, moderate if

4 it required intervention (e.g. oral analgesic for headache), and severe if it required hospitalization.

5 There were no severe adverse events reported in either group at any time point. No participants

6 experienced intraoperative complications.

$7 \quad{ }^{*}$ Two participants in the END group experienced PACU complications (non-cardiac chest pain,

8 which resolved in PACU and wheezing, which resolved with albuterol administered in PACU).

9 †Two participants in the NRT group experienced self-reported postoperative complications within

10 30-days (both wound complications), while 5 participants in the END group had postoperative

11 complications (2 wound-related, 2 bladder-related and 1 respiratory).

$12{ }^{\ddagger}$ All complications assessed by chart-review were wound-related in both NRT and END groups. 
13 NRT = nicotine replacement therapy. END = electronic nicotine delivery $(\mathrm{e}$-cigarette $)$. PACU = post14 anesthesia care unit. 


\section{Table 5 (on next page)}

Participant usage and satisfaction

p-value form Fisher's exact test for product usage and Wilcoxon ranksum test for all other values. Likert scale was used for agreement ( $1=$ strongly disagree, $2=$ disagree, $3=$ disagree somewhat, $4=$ neither agree nor disagree, $5=$ agree somewhat, $6=$ agree, $7=$ strongly agree). NRT = nicotine replacement therapy. END = electronic nicotine delivery (ecigarette). IQR = interquartile range *Asked about use while supplies lasted (e.g. considered the participant to have used the product daily or most days if they used the product until they ran out) 
1

Table 4. Participant usage and satisfaction

\begin{tabular}{|c|c|c|c|}
\hline Question & NRT Group & END Group & $p$ \\
\hline Day of surgery & $(n=10)$ & $(n=19)$ & \\
\hline Used product daily or most days, $\mathrm{n}(\%)$ & $5(50 \%)$ & $11(58 \%)$ & 0.71 \\
\hline \multicolumn{4}{|l|}{ Agreement (Likert scale 1-7) with } \\
\hline $\begin{array}{l}\text { "The product is helpful for quitting smoking," } \\
\text { median [IQR] } \\
\text { "I was satisfied with the product to help with }\end{array}$ & $5[3-7]$ & $6[4-7]$ & 0.59 \\
\hline $\begin{array}{l}\text { quitting," } \\
\text { median }[\mathrm{IQR}]\end{array}$ & $5[3-6]$ & $6[4-6]$ & 0.71 \\
\hline $\begin{array}{l}\text { "I would recommend the product to someone } \\
\text { interested in quitting smoking," median [IQR] }\end{array}$ & $6[5-7]$ & $6[6-7]$ & 0.73 \\
\hline 8-weeks after randomization & $(n=9)$ & $(n=20)$ & \\
\hline Used product daily or most days ${ }^{*}, \mathrm{n}(\%)$ & $6(67 \%)$ & $16(80 \%)$ & 0.64 \\
\hline \multicolumn{4}{|l|}{ Agreement (Likert scale 1-7) with } \\
\hline $\begin{array}{l}\text { "The product is helpful for quitting smoking," } \\
\text { median [IQR] } \\
\text { "I was satisfied with the product to help with }\end{array}$ & $5[3-7]$ & $6[4-7]$ & 0.79 \\
\hline $\begin{array}{l}\text { quitting," } \\
\text { median }[\mathrm{IQR}]\end{array}$ & $5[3-6]$ & $5.5[2.5-7]$ & 0.67 \\
\hline $\begin{array}{l}\text { "I would recommend the product to someone } \\
\text { interested in quitting smoking," median [IQR] }\end{array}$ & 7 [6-7] & $6[5-7]$ & 0.46 \\
\hline
\end{tabular}

2 p-value from Fisher's exact test for product usage and Wilcoxon ranksum test for all other

3 values. Likert scale was used for agreement ( $1=$ strongly disagree, $2=$ disagree, $3=$

4 disagree somewhat, $4=$ neither agree nor disagree, $5=$ agree somewhat, $6=$ agree, $7=$

5 strongly agree).

6 NRT = nicotine replacement therapy. END = electronic nicotine delivery (e-cigarette). IQR

7 = interquartile range

$8{ }^{*}$ Asked about use while supplies lasted (e.g. considered the participant to have used the

9 product daily or most days if they used the product until they ran out) 


\section{Table 6(on next page)}

\section{Specific Adverse Events}

Values are $n$ (percentage). p-values from Fisher's exact test. Events were considered to have occurred if patient reported the symptom at any time point assessed (day of surgery, 30days postoperatively and 8-weeks follow-up). Patients were also given a phone number to call if they experienced side-effects; there were no calls. No participants reported hospitalization unrelated to surgery. No participants reported pneumonia. Other adverse events in the NRT group included: irritable mood, patch not sticking properly, increased cravings, jitteriness, diarrhea, dry mouth, anxiety, sleepiness. Other adverse events in the END group included: slight wheezing, productive cough, choking sensation, poor appetite, burning sensation, burned lip. NRT = nicotine replacement therapy. END = electronic nicotine delivery (e-cigarette). 
1

Table 6. Specific Adverse Events

\begin{tabular}{lccc}
\hline Adverse event & $\begin{array}{c}\text { NRT Group } \\
(\mathbf{n = 1 0 )}\end{array}$ & $\begin{array}{c}\text { END Group } \\
(\mathbf{n = 2 0 )}\end{array}$ & $\boldsymbol{p}$ \\
\hline Headache & $4(40 \%)$ & $4(20 \%)$ & 0.38 \\
Nausea & $1(10 \%)$ & $5(25 \%)$ & 0.63 \\
Dry cough (persistent) & $0(0 \%)$ & $2(10 \%)$ & 0.54 \\
Dry cough (intermittent) & $1(10 \%)$ & $6(30 \%)$ & 0.37 \\
Palpitations & $2(20 \%)$ & $0(0 \%)$ & 0.10 \\
Throat irritation & $3(30 \%)$ & $5(25 \%)$ & 1.0 \\
Skin irritation & $3(30 \%)$ & $2(10 \%)$ & 0.30 \\
Other & $6(60 \%)$ & $7(35 \%)$ & 0.26 \\
\hline
\end{tabular}

2 Values are $n$ (percentage). p-values from Fisher's exact test. Events were considered to have

3 occurred if patient reported the symptom at any time point assessed (day of surgery, 30-days

4 postoperatively and 8-weeks follow-up). Patients were also given a phone number to call if they

5 experienced side-effects; there were no calls. No participants reported hospitalization unrelated to

6 surgery. No participants reported pneumonia. Other adverse events in the NRT group included:

7 irritable mood, patch not sticking properly, increased cravings, jitteriness, diarrhea, dry mouth,

8 anxiety, sleepiness. Other adverse events in the END group included: slight wheezing, productive

9 cough, choking sensation, poor appetite, burning sensation, burned lip.

10 NRT $=$ nicotine replacement therapy. END = electronic nicotine delivery (e-cigarette).

11 


\section{Table 7 (on next page)}

California Smokers' Helpline Services

Values are $n$ (percentage). p-values from Fisher's exact test. *All participants had a webbased referral confirmed by the study team. However, the California Smokers' Helpline did not locate the referral for 2 patients in the NRT group and 1 patient in the END group. 
1

\begin{tabular}{lccc}
\hline \multicolumn{4}{l}{ Table 7. California Smokers' Helpline Services } \\
\hline Service & $\begin{array}{c}\text { NRT Group } \\
(\mathbf{n}=\mathbf{1 0})\end{array}$ & $\begin{array}{c}\text { END Group } \\
(\mathbf{n}=\mathbf{2 0})\end{array}$ & $\boldsymbol{p}$ \\
\hline Referral received* & $8(80 \%)$ & $19(95 \%)$ & 0.25 \\
No contact made & $3(30 \%)$ & $8(40 \%)$ & 0.70 \\
Reached & $5(50 \%)$ & $11(55 \%)$ & 1.0 \\
Received counseling & $0(30 \%)$ & $3(15 \%)$ & 0.53 \\
Received materials & $2(20 \%)$ & $1(5 \%)$ & 0.25 \\
Refused service & $3(30 \%)$ & $7(35 \%)$ & 1.0 \\
\hline
\end{tabular}

2 Values are $n$ (percentage). p-values from Fisher's exact test.

$3 \quad$ *All participants had a web-based referral confirmed by the study team. However, the California

4 Smokers' Helpline did not locate the referral for 2 patients in the NRT group and 1 patient in the 5 END group.

6 